\section{Klaus Biemann}

${ }^{1}$ Klaus Biemann's Account and Perspective that headlines this issue of J. Am. Soc. Mass Spectrom. is filled with interesting reminiscences and serves as a fitting personal summary of his career in organic and biological mass spectrometry, starting in the 1950s with his efforts in natural-product structure determination. Professor Biemann's long and illustrious history of pioneering the application of mass spectrometry to the analysis of a wide variety of important organic and biological compounds is well known, but few may be aware of his significant contribution to the exploration of the planet Mars.

In the late 1960s, the National Aeronautics and Space Administration (NASA) planned a mission to the surface of Mars, the Viking Project, to investigate its atmosphere and search for the presence of life. In addition to a series of life detection experiments proposed by the biological community, NASA also wanted to include an experiment to detect organic compounds to deal with the possibility that non-biogenic organic compounds might be present on the Martian surface. While this may seem far-fetched, it had been shown in the 1960s that organic compounds were indigenous to carbonaceous chondrite meteorites that had fallen to Earth. To accomplish the task of searching for organic compounds on the Martian surface, NASA chose a miniature gas chromatograph/mass spectrometer (GC/ MS) system proposed by Prof. Biemann as part of the payload of the Mars lander (Figure 1 is a photograph of Biemann with the instrument. The bolo tie is apparently de rigueur attire for those who work in the Southwest).

The challenges of accomplishing this task were truly formidable. The technology of combining the gas chromatograph with a mass spectrometer was quite new. To add to the difficulty, the instrument had severe weight and power consumption constraints. Finally, an instrument analysis protocol had to be devised several years prior to the arrival of the Viking spacecraft on Mars in 1976 without any knowledge of the types of organic compounds that might be present, if any.

Despite these difficult challenges, the Mars lander GC/MS experiment was an outstanding success. The instrument operated as planned, performing the experimental protocol flawlessly on command from Earth. The result was something of a disappointment because no organic compounds were detected in the Martian soil samples taken by two Mars landers. However, this null result was unequivocal because traces of the solvents used in cleaning the instrument components before the spacecraft was launched-and detected during a mid-course blank run-clearly showed up in the experiments on Mars. Furthermore, the negative results

Published online September 17, 2002

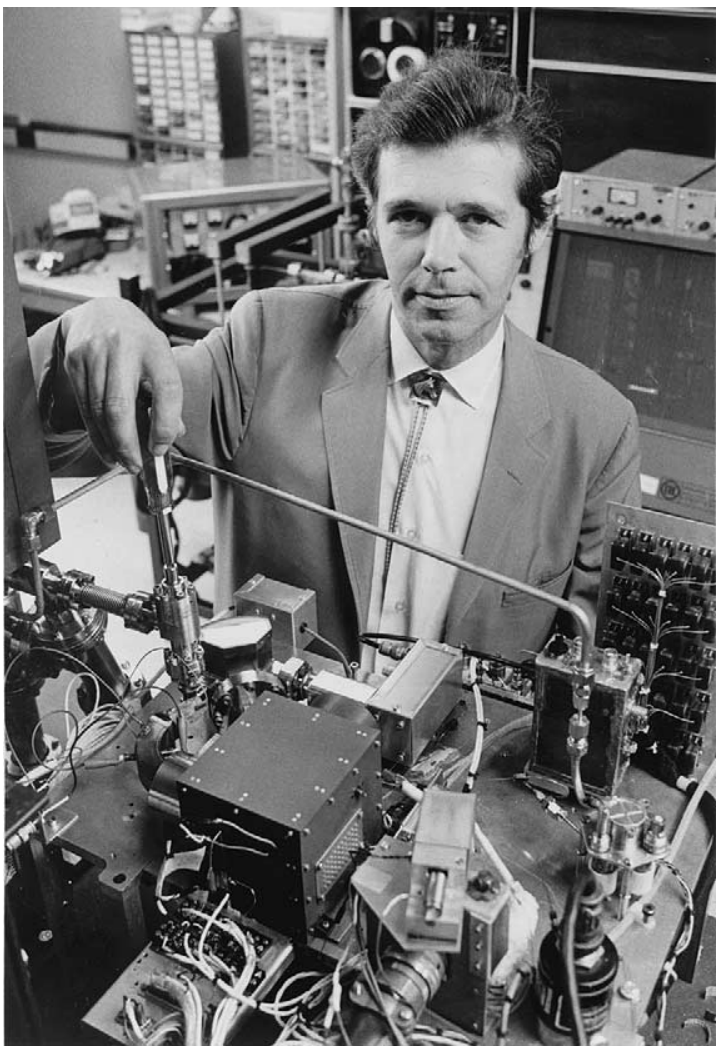

Figure 1. Professor Biemann in 1975 with the science test unit of the GC/MS used on the Mars lander.

for the presence of organic compounds were in agreement with the negative results returned from the three Project Viking biology experiments.

The mass spectrometer of the GC/MS system was then switched to a special atmospheric sampling system for removing carbon monoxide and carbon dioxide, which made up the bulk of the Martian atmosphere, thus concentrating the other gases. This experiment enabled the determination of nitrogen and four noble gases in the Martian atmosphere down to the sub part-per-million level. Further, the mass spectrometer provided information about the isotope ratios of carbon, nitrogen, oxygen, argon, and xenon at the surface of Mars, information valuable to planetologists in determining the early history of the planet.

The success of the surface science mass spectrometer on the Viking Project was due in no small part to the expert team assembled and led by Prof. Biemann. He brought the same scientific discipline and execution to Project Viking that was characteristic of his long career in the study of organic compounds here on Earth.

Michael A. Grayson Washington University at St. Louis ASMS Archivist 